\title{
Response class interference in STM
}

\author{
DELOS D. WICKENS and SHERYL A. CAMMARATA \\ Colorado State University, Fort Collins, Colorado
}

\begin{abstract}
In this experiment, we investigated why the performance decline across a series of BrownPeterson trials, using a constant short $(30-\mathrm{sec})$ intertrial interval (ITI), is reversed by increasing the ITI on the subsequent trial. One interpretation is that the increased time serves as a marker that emphasizes the succeeding trial and its information, resulting in better recall. In the present experiment, we increased the ITI by presenting a word matrix to be read during the added time interval. For different groups the matrix was of the same semantic material as the memory triads, was quite similar, or was quite different. The amount of improvement on the next trial was a function of the degree of semantic disparity between the matrices and the memory triads. Mere presentation of the matrix was not sufficient to influence performance on the subsequent trial, but the semantic nature of the matrices did exert such an influence.
\end{abstract}

If, in the Brown-Peterson paradigm (Brown, 1958; L. R. Peterson \& M. J. Peterson, 1959), triads of items from the same semantic class are presented at a constant intertrial interval (ITI) of $30 \mathrm{sec}$, performance will decline progressively across the first four or five trials and will remain at about this level across succeeding trials. This is generally assumed to result from the buildup of proactive inhibition (PI). An improvement in performance can be obtained by two procedural changes. One is to change the semantic class of the triads (Wickens, 1970; Wickens, Born, \& Allen, 1963), a procedure usually termed release from PI. The other is to lengthen the intertrial interval (Kincaid \& Wickens, 1970; Nield, 1968; Peterson \& Gentile, 1965).

The basis for the present research stems from a reaction to Nield's (1968) study. In Nield's study, five trials were administered at an ITI of $16 \mathrm{sec}$; the materials were consonant trigrams (CCC), and the distractor task was color naming. After five trials, for one group (Rest), the experimenter simply held his hand over the lens for a varying period of time (the subjects had been prewarned that this interruption would occur); for another group (Color Naming), matrices of colors were presented for varying periods of time; for the third group (Experimental), the Brown-Peterson (B-P) task was continued but with a different class of materials, namely three-digit numbers (NNN). For one set of these three groups, this interval was $16 \mathrm{sec}$; for another set, $48 \mathrm{sec}$; and for the final set, $80 \mathrm{sec}$. Then all groups were given a final trial on a CCC trigram. Thus, the usual ITI between the fifth and sixth trials was increased by 16,48 , or $80 \mathrm{sec}$. The subjects in all groups were warned to expect a brief interruption or change in procedure at some time. The increased ITI length had only a small nonsignificant effect for the 16sec group, but performance increased by about $45 \%$ for

This research was conducted in the Experimental Psychological Laboratories at this institution. The authors' address is: Department of Psychology, Colorado State University, Fort Collins, CO 80523. the 48 -sec group and $60 \%$ for the 80 -sec group. There was, however, no significant effect of the type of interpolated activity; all three groups had almost identical scores, which increased at the successive intervals.

The senior author of this article had presented this research at a meeting of a group of psychologists with strong interests in verbal learning and memory. One of the members suggested that the simple interpolation of a matrix for the subject to process between the fifth and sixth trials would produce a performance increment; this would occur because the interpolation, as a unique event, would function as a marker to facilitate performance of the upcoming trial.

In an unpublished experiment, Nield and Wickens decided to test this interpretation using two kinds of matrices, one composed of consonants and the other composed of digits. One of these matrices was inserted for $60 \mathrm{sec}$ between Trials 3 and 4 . The Brown-Peterson materials were either CCC or NNN. The distractor activity was color naming. The matrices were either of the same class as the B-P task or of the other class. The trial after the matrix condition was always of the same class as that of the first three trials, that is, CCCs for half of the subjects and NNNs for the other half.

The results of the experiment were very clear: if the materials of the B-P trials were of the same class as the matrix, no improvement in recall occurred on the postmatrix trial. However, if the matrix and B-P materials differed (digits for the consonant group and consonants for the digit group), then a large increment in recall was found on the postmatrix trial. This was true for both sequences, consonants to digits or digits to consonants. Obviously, the results do not support a simple marker interpretation; the content of the matrix is important.

The present experiment extended this line of inquiry, using words in order to produce finer semantic gradations of difference than those afforded by consonants versus digits. With respect to the word classes used, this experiment is modeled after one reported by Wickens, Dalez- 
man, and Eggemeier (1976), which tested the role of the number of attributes shared by the control (nonshift) group and the experimental (shift) group. In that experiment, the taxonomic category used on the final trial for all groups was fruits, but the groups differed in the class of materials experienced during the first three trials. For one group, the category employed was vegetables, since vegetables share two attributes with fruits: both grow from the ground and are edible. The items in the categories for the two other groups each shared only one attribute with fruits. One category was meats, which are edible but do not grow from the ground, and the other was flowers, which are not edible but do grow from the ground. Items in the final category, professions, share neither of these attributes with fruits.

In the experiment of Wickens et al. (1976), the degree of release from PI closely conformed to the attributes-incommon prediction. The Tukey $A$ test showed no significant differences among the five groups across the first three trials. On Trial 4, the critical postmatrix trial, using a .05 level, the following order (from the highest group to the lowest group) was obtained: professions $>$ flowers $=$ meats $>$ vegetables $>$ fruits.

The strategy used in the present experiment was to combine the matrix manipulation used in Nield and Wickens's unpublished experiment and the manipulation of the degree of similarity of matrix and target (to-beremembered, or TBR) material used in Wickens et al.'s (1976) study. Quite obviously, the prediction was that the more semantically dissimilar the matrix was to the target material, the better the performance on the postmatrix trial.

\section{METHOD}

\section{Subjects}

The subjects were 96 undergraduate students who received experimental participation credit in introductory psychology classes at Colorado State University. The subjects were randomly assigned to four different groups (two experimental and two control) in a rotating order of their appearance. There were 24 subjects per group, each subject participating singly in a quiet room.

\section{Apparatus and Materials}

A Kodak carousel slide projector was used for presentation of various word trigram trials and interpolated matrices. The duration of each slide presentation was controlled by a tape timing device. Category norms were selected from lists composed by Battig and Montague (1969). The categories used for trigram testing were fruits in Part 1 and insects in Part 2. The specific slides used were as follows: fruits-banana, peach, apple; tangerine, cherry, grape; grapefruit, apricot, lemon; pineapple, raspberry, melon; insects-fly, gnat, louse; bee, tick, mosquito; ant, wasp, cricket; moth, roach, beetle.

Matrices were composed of words drawn from the following categories: same (fruits, insects), similar (vegetables, fish), or different (occupations, cities). The fourth group of subjects received no matrix; they simply rested for the designated period of time.

\section{Procedure}

All subjects were tested on both Parts 1 and 2, the parts being defined by the B-P triads, whether insects or fruits. In each part of the experiment, the matrices were either same, similar, or different. The orders of trigram presentation and matrix presentation were completely counterbalanced. A standard B-P and release-from-PI procedure was employed. A green slide was presented for $1 \mathrm{sec}$ as an indication to the subject that he/she would be working with trigrams (as opposed to a red slide, indicating a matrix task). Following the green slide was a 1sec presentation of an asterisk as a ready signal. This was followed by a 2-sec presentation of the first fruit trigram, which the subject was instructed to read aloud as quickly and accurately as possible. The fruit trigram was replaced by a three-digit number from which the subject was required to count backward by threes for $18 \mathrm{sec}$. A question mark then appeared, indicating that the subject should attempt to recall the trigram. This procedure was repeated for three trials and then a red slide appeared for $1 \mathrm{sec}$. At this time the matrix task was presented, in the form of five slides containing eight words each. The subject was given $8 \mathrm{sec}$ per slide to accomplish the task. Subjects were aware that they should only read the matrix and that they would not at any time be asked to recall those words. After the subject read the matrix aloud, a green slide was presented again, indicating the return to Trigram Trial 4 (the test trial). The subjects were given a brief rest period between Part 1 testing and Part 2 testing.

The relationship between the semantic class of the triads and the class of the matrix was the same for both Parts 1 and 2 within each experimental group of same, similar, or different material.

The number of words remembered on each of the trigram trials was recorded and 1 point was given for recall of all three words in the correct order. The data was collapsed over the two parts, making 8 points the maximum number possible per trial. The major matters of interest here are, of course, performance on the critical postmatrix trial and the degree of similarity of the matrix to the trigram class.

\section{RESULTS}

The mean performance of all groups on all trials is presented in Figure 1. Performance is represented as percent correct. For example, the mean percentage of 100 represents a perfect score of 8 .

These results were statistically evaluated by analysis of variance (ANOVA) across the first three trials and again across the fourth trial, all four groups being included in each test. An ANOVA showed no significant difference among groups across the first three trials $[F(3,92)=.51$, $p<.05$ ]. However, a marked trials effect in all groups

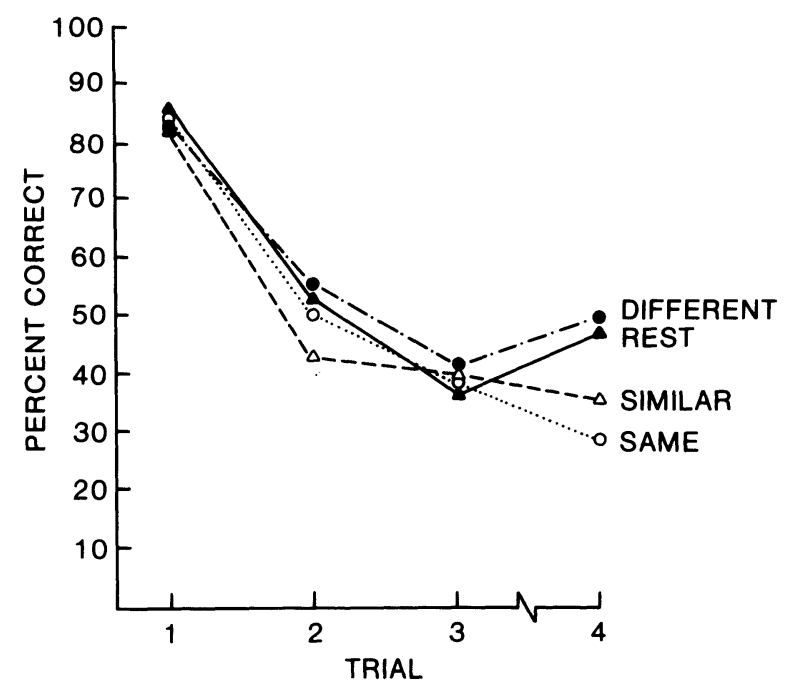

Figure 1. Mean percent correct recall for all groups across the four trials. The marker between Trials 3 and 4 indicates that the intertrial interval was increased to include the matrix task or rest. 
was significant $[F(2,184)=157.00, p<.05]$, indicating that the subjects' ability to recall material decreased across the first three trials. A significant difference among groups was seen on Trial $4[F(3,92)=3.22, p<.05]$, and the Tukey $A$ test showed the following ordering: different $=$ rest $>$ similar $=$ same. In other words, there is no significant difference between the groups different and rest or the groups similar and same; however, the former two and latter two groups differ significantly at the .05 level.

\section{DISCUSSION}

The primary purpose of this research was to test the hypothesis that release from PI (Wickens, 1970) is produced by some dramatic or marker event which differentiates the "release trial" from the preceding few trials, the change serving to emphasize the memory set of that trial so that it becomes especially well remembered. An unpublished study by Nield and Wickens used two classes of materials for the TBR sets and matrices (consonants and digits) and found release only if the matrix and TBR sets differed in class. The current research extended this research using meaningful material.

The outcomes of the current study are in conceptual agreement with Nield and Wickens's unpublished research. A matrix of the same semantic class resulted in a continued decline in performance after the interpolated $45 \mathrm{sec}$ of matrix processing, and a matrix of a highly similar semantic class seemed to maintain inhibition at the level of the last trial. However, the different matrix condition and the rest condition both permitted a dissipation of the PI generated during the first three trials. It is apparent that the mere interpolation of a matrix is not sufficient to reduce inhibition; inhibition depends on the relationship between the materials composing the matrix and those in the B-P task.

The reader should be reminded that the specific words in the matrices were not used to compose the TBR items, hence the use of the term response class in the title. The subjects were told they would not be asked to recall the matrix items. The matrix and the TBR activities were differentiated by colored slides informing the subjects which activity would occur. A break between sessions also reinforced this differentiation. Apparently, simply perceiving and verbalizing words of the same or similar class as the TBR category will maintain the inhibition generated by the three consecutive B-P trials.

The results of this experimental paradigm bear a similarity to those sometimes obtained from part-set cuing (Nickerson, 1984; Slamecka, 1968). In the free-recall aspect of part-set cuing research, the experimenter may present to a group of subjects a random half of the words in the list at the beginning of the recall period and then compare this group's performance with that of a control group given no cuing words. The comparison is based only on the words not included in the half-list that was presented by the experimenter. In several instances the cuing has resulted in poorer performance by the cued group than by the control group. In his review, Nickerson (1984) concluded that this field remains something of an enigma in memory research. Neither of these two lines of research - release from PI or part-set cuing-explains the other, but there does appear to be a conceptual overlap between the two paradigms. The overlap is, of course, the inhibition produced by merely processing other verbally related materials.

\section{REFERENCES}

Battig, W. F., \& Montague, W. E. (1969). Category items for verbal items in 56 categories: A replication of the Connecticut category norms. Journal of Experimental Psychology Monographs, 80(3, Pt. 2).

BRown, J. (1958). Some tests of the decay theory of immediate memory. Quarterly Journal of Experimental Psychology, 58, 193-198.

KINCAID, J. P., \& WICKENS, D. D. (1970). Temporal gradient of release from proactive inhibition. Journal of Experimental Psychology, 86, 313-316.

NiCKERSON, R. S. (1984). Retrieval inhibition from part-set cuing: A persisting enigma in memory research. Memory \& Cognition, 12, 531-551.

NIELD, A. F. (1968). The effects of time and activity on the dissipation of proactive inhibition in short-term memory. Unpublished master's thesis, Ohio State University, Columbus.

Peterson, L. R., \& Gentile, A. (1965). Proactive interference as a function of the time between tests. Journal of Experimental Psychology, 70, 473-478.

Peterson, L. R., \& Peterson, M. J. (1959). Short-term retention of individual verbal items. Journal of Experimental Psychology, 58, 193-198.

SLAMECKA, N. J. (1968). An examination of trace storage in free recall. Journal of Experimental Pscyhology, 76, 504-513.

WICKENS, D. D. (1970). Encoding categories of words: An empirical approach to meaning. Psychological Review, 77, 1-15.

Wickens, D. D., Born, D. G., \& Allen, C. K. (1963). Proactive inhibition and item similarity in short-term memory. Journal of Verbal Learning \& Verbal Behavior, 2, 440-445.

Wickens, D. D., Dalezman, R. E., \& EgGemeier, F. T. (1976). Multiple encoding of word attributes in memory. Memory \& Cognition, 4, 307-310.

(Manuscript received March 31, 1986.) 